\title{
Efectividad del Uso de Placas Palatinas y de la Estimulación Orofacial en el Desarrollo Oral en Niños con Síndrome de Down
}

\author{
MARÍA JOSÉ PADRÓ S. ${ }^{1}$, EVELYN BARRAZA V. ${ }^{1}$, CONSTANZA BRÜCHER S. ${ }^{1}$, \\ ERIKA CONCHA T. ${ }^{1}$, VERÓNICA DELGADO ${ }^{2}$ \\ 1. Cirujano Dentista, Cátedra de Odontopediatría, Universidad de Valparaíso. \\ 2. Médico Fisiatra Hospital Carlos Van Buren de Valparaíso.
}

\begin{abstract}
Effectiveness of the Use of Palate Plates and Orofacial Stimulation in the Oral Development of Children with Down's Syndrome
\end{abstract}

Down's Syndrome children shown multiple alterations, among them marked general hypotony. While this produces some basic difficulties, at the orofacial level it generates a characterisitic expression marked by open mouth, hypotonic tongue that rests upon the lower lip, and great salivation. To ameliorate this, an orofacial regulation therapy was initiated, to stimulate this musculature, strengthen it and mold it during development. Objective: To observe the effect of stimulating palate plates, and orofacial exercises in the function and tonicity of children with Down's syndrome. Patients and Methods: Five children, 10 to 21 months old, with Down's Syndrome, were treated through orofacial regulation for 20 to 30 minutes, 4 times a day for 2 months (palate plates and orofacial stimulation exercises). Pre and post treatment evaluations were made and a photographic and video evaluation was registered weekly. Results and Conclusions: Therapy decreased lingual protrusion and increased buccal closure. This is not thought of as real tonicity change since that statement would require a long term and wider area study to determine effectiveness and applicability.

(Key words: Orofacial stimulation, palate plates, Down's Syndrome).

Rev Chil Pediatr 2010; 81 (1): 46-52

\section{RESUMEN}

Introducción: Se sabe que los pacientes con Síndrome de Down presentan múltiples alteraciones, entre ellas marcada hipotonía general lo que dificulta las funciones básicas y a nivel orofacial genera una expresión característica de boca abierta, lengua hipotónica descansando sobre el labio inferior y gran salivación. En respuesta a esto se creó la terapia de regulación orofacial que estimula la musculatura orofacial, para fortalecerla y modelarla durante el desarrollo. Objetivo: Observar los efectos de las placas palatinas estimulantes y de los ejercicios orofaciales, en la función y tonicidad de la musculatura orofacial de niños con Síndrome de Down.

Trabajo recibido el 08 de mayo de 2009, devuelto para corregir el 01 de junio de 2009, segunda versión el 06 de agosto de 2009, aceptado para publicación el 31 de agosto de 2009.

Correspondencia a:

María José Padró Silva

E-mail: dra.padro@gmail.com 
Pacientes y Métodos: seleccionamos 5 niños con síndrome de Down, entre 10 y 21 meses de edad, se les aplicó la terapia de regulación orofacial durante 2 meses con uso de 20 a 30 min por 4 veces al día (placas palatinas estimulantes y ejercicios de estimulación orofacial). Se realizaron evaluaciones pre y post tratamiento, y controles semanales con registro fotográfico y video. Resultados y Conclusiones: La terapia aumentó el cierre bucal y disminuyó la protrusión lingual. Sin embargo, no se observaron cambios "reales" en la tonicidad muscular, ya que su evaluación requiere estudios a largo plazo y de un universo mayor, para determinar la efectividad de la técnica y masificar su aplicación.

(Palabras clave: Estimulación orofacial, placas palatinas, Síndrome de Down).

Rev Chil Pediatr 2010; 81 (1): 46-52

\section{Introducción}

En la cavidad oral de un paciente con Síndrome de Down se ven afectadas sus estructuras, y por consiguiente sus funciones, es común apreciar un paladar estrecho y profundo, alteraciones en la dentición, una lengua seudomacroglósica, labio inferior evertido y un pobre cierre bucal. Todas estas últimas características son provocadas por la marcada hipotonía presente en los pacientes que sufren este síndrome, y son estas alteraciones las que además de impedir o dificultar algunas funciones básicas del ser humano tales como la masticación, la deglución y la fonación entre otras, no permiten un normal desarrollo del individuo y los marca con una expresión característica; boca abierta, lengua descansando hipotónica sobre un labio inferior evertido y una gran salivación.

Buscando tratar estas alteraciones y poniendo especial énfasis en la zona orofacial, en la década del 70 el médico Argentino Castillo-Morales, introdujo en Europa un método para la rehabilitación neuromotora de niños discapacitados ${ }^{1}$, universo en el que entran los niños con Síndrome de Down. Este método presentado por Castillo-Morales incluye una terapia de regulación orofacial, la que está conformada por ejercicios de estimulación orofacial, sumada a un dispositivo para el entrenamiento de la lengua y los músculos peribucales: la "placa palatina", la cual mejora la función orofacial y la apariencia facial de los niños con Síndrome de Down ${ }^{2-5}$.

Estos estudios nos llevaron a realizar un estudio piloto cuyo objetivo era observar los efectos de las placas palatinas estimulantes y de los ejercicios orofaciales en la función y tonicidad muscular en niños con Síndrome de Down.

\section{Pacientes y Método}

Para la selección de los pacientes se establecieron criterios de inclusión. Los niños debían tener hipotonía evidente al examen clínico, ser menores de dos años, no tener patologías sistémicas asociadas, estar asistiendo a centros de rehabilitación infantil y sus padres debían aceptar las condiciones del estudio y firmar un consentimiento informado. Se obtuvo una muestra de 5 pacientes con Síndrome de Down, tres hombres y dos mujeres, con un promedio de edad de 15 meses. A todos los pacientes se les colocó placas palatinas y ejercicios de estimulación orofacial vibratorios por un período de dos meses, tiempo en el que recibieron controles semanales y cuatro evaluaciones. El seguimiento de estos dos meses fue registrado mediante videos y fotografías

Para fabricar las placas palatinas, previamente se tomaron impresiones. Por la edad de los pacientes y el escaso desarrollo maxilar las cubetas se fabricaron especialmente para el estudio. La impresión fue tomada con silicona pesada Speedex. El niño debía sentarse sobre las piernas de su madre y el operador a su derecha. Una vez ubicada la cubeta, se realizaban movimientos de tracción del labio y de las mejillas para una correcta impresión de los flancos funcionales, de la musculatura y freni1los (figuras 1-3). 
Se obtuvieron modelos de yeso extraduro sobre los que se confeccionaron las placas. La base fue hecha por medio de un estampado de acetato (figura 4) y sobre ella se pusieron dos tipos de botones estimulantes con formas de medias esferas, uno a nivel de la línea media del paladar y los otros se distribuyeron en la porción que cubre el reborde en la zona anterior en relación al labio (figura 5).

Un médico fisiatra fue el encargado de realizar la evaluación inicial previa a la instalación de las placas y de instruir a los padres en cuanto a los ejercicios a realizar. Los ejercicios fueron indicados en número de 10 veces antes y después del uso de la placa (figura 6). Se in-

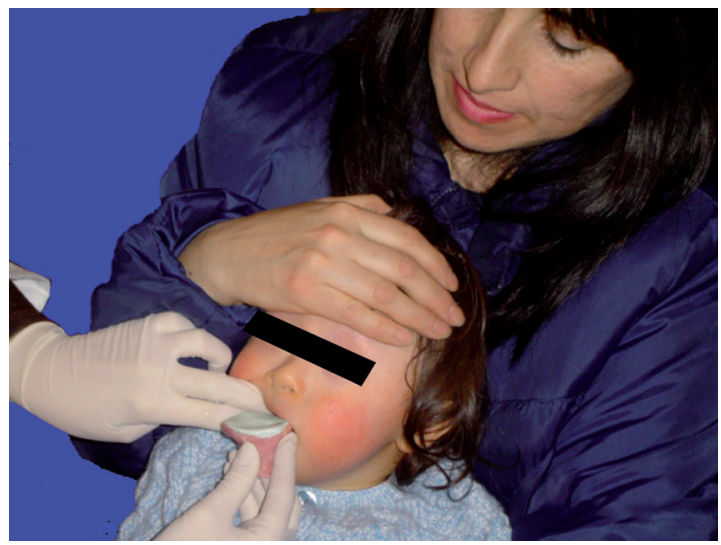

Figura 1. Toma de impresión con silicona pesada.

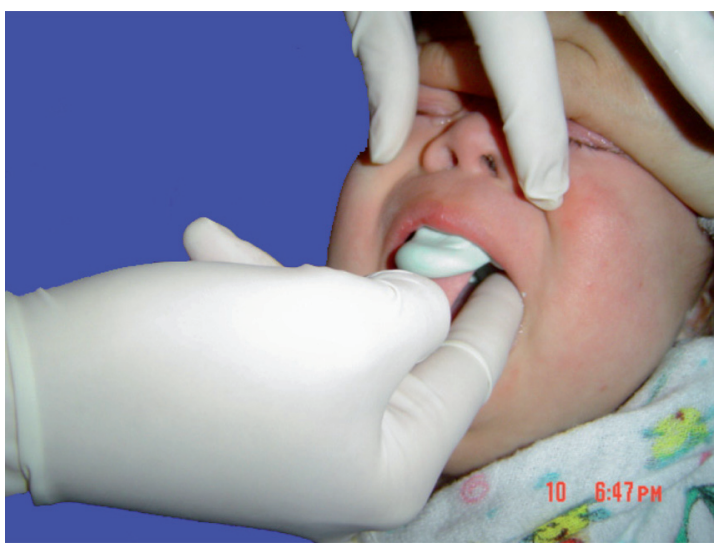

Figura 3. Toma de impresión con silicona pesada. dicó usar la placa 4 veces al día por períodos de entre 15-30 minutos.

Se evaluaron 5 parámetros, a los que se les asignó puntaje. Los parámetros fueron: a) tono muscular del labio superior $(3=$ tono normal, $2=$ hipotonía leve, $1=$ hipotonía moderada, $0=$ hipotonía severa); b) tono muscular de la lengua $(3=$ tono normal, $2=$ hipotonía leve, $1=$ hipotonía moderada, $0=$ hipotonía severa); c) postura labial ( $3=$ boca completamente cerrada, $2=$ apertura bucal leve, $1=$ apertura bucal moderada, $0=$ boca ampliamente abierta); d) posición de la lengua $(3=$ completamente dentro de la boca, $2=$ posición interdentaria, $1=$ posición interlabial, $0=$ posicionada más

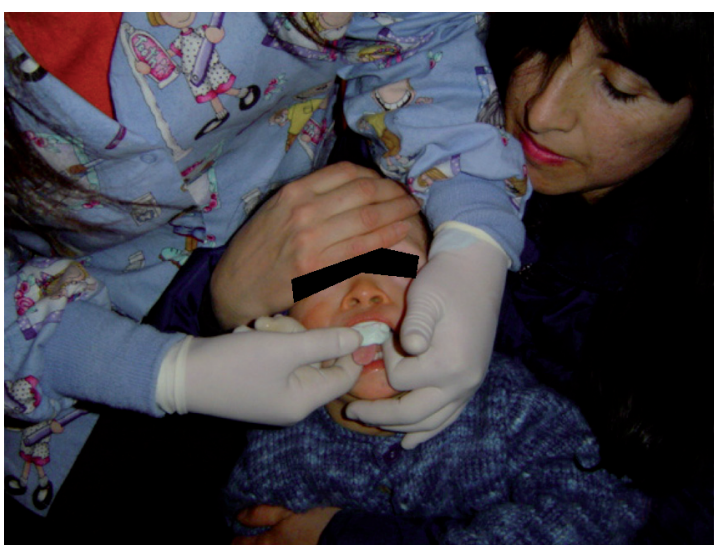

Figura 2. Toma de impresión con silicona pesada.

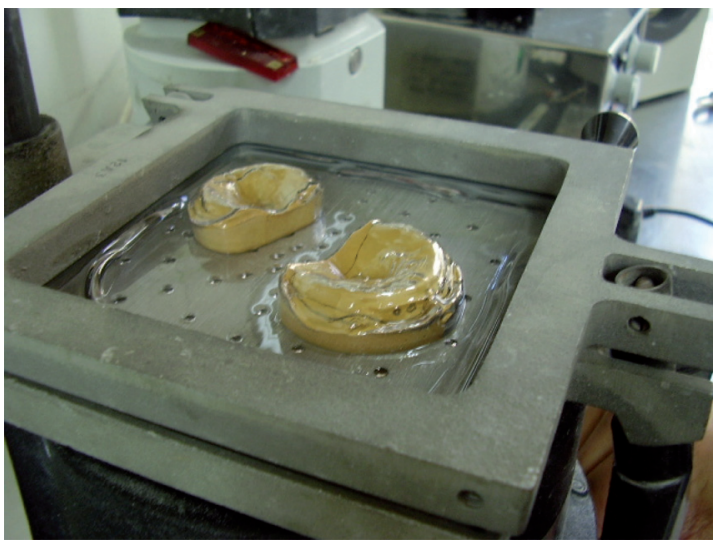

Figura 4. Modelos de yeso extraduro. 


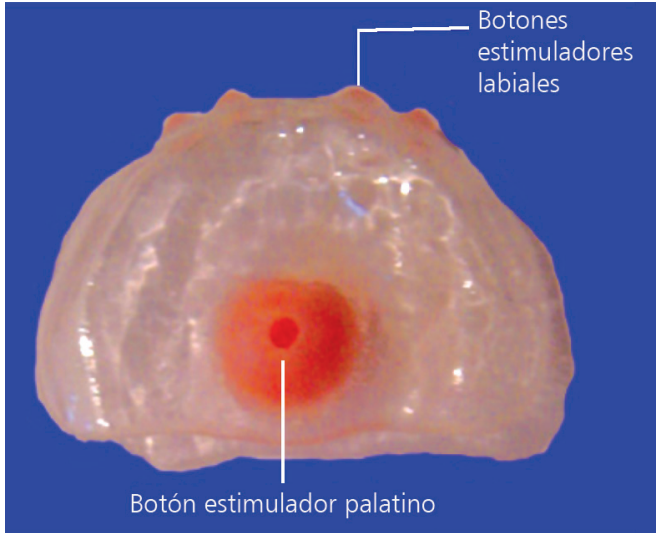

Figura 5. Placa palatina de acetato con botones de estimulación.

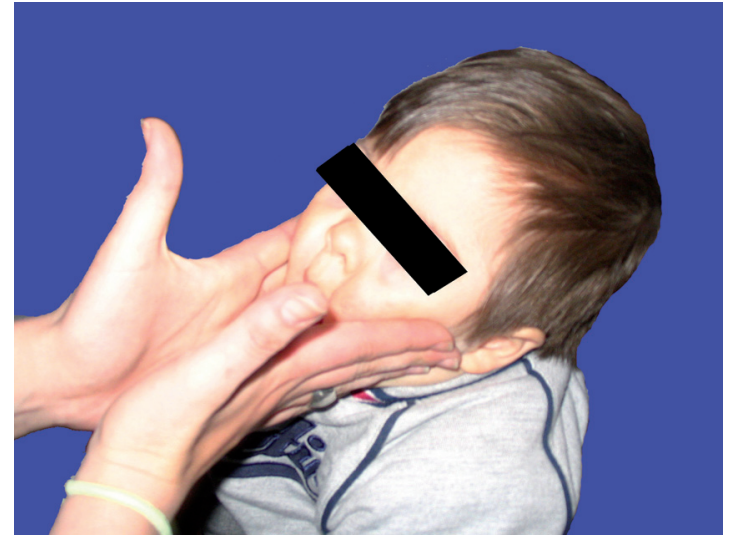

Figura 6. Ejercicio de estimulación orofacial.
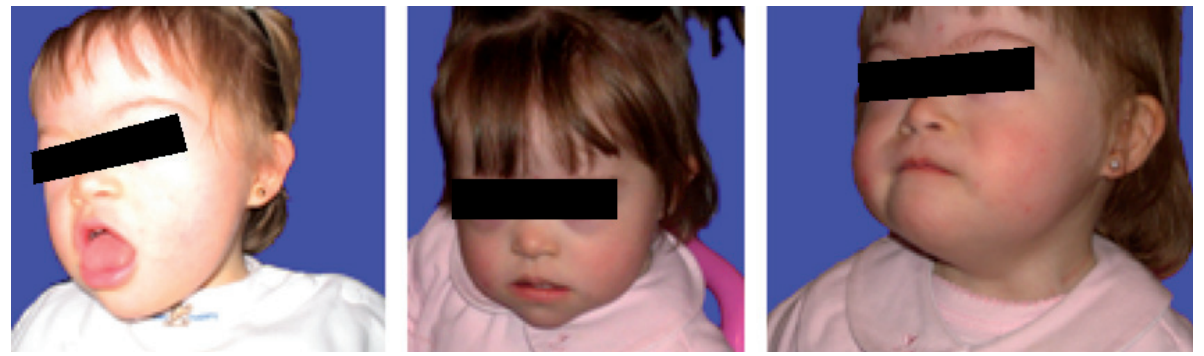

Figura 7. Evolución en paciente con uso de placa palatina y ejercicios orofaciales.
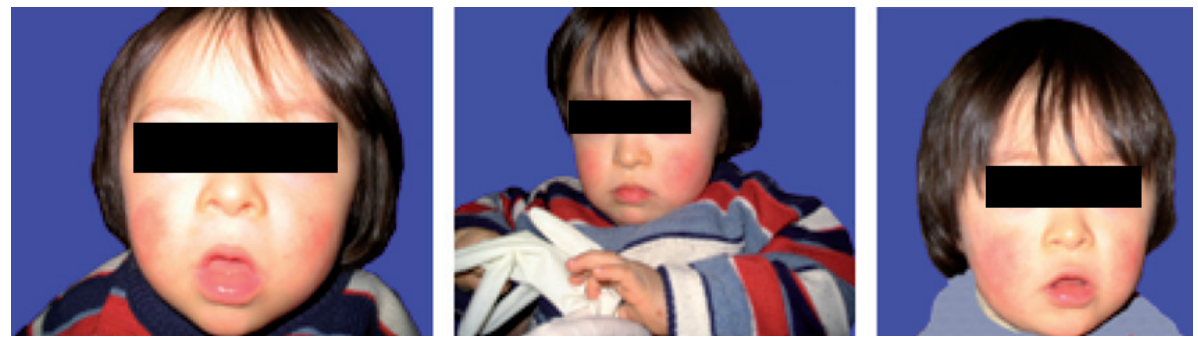

Figura 8. Evolución en paciente con uso de placa palatina y ejercicios orofaciales.

allá de los labios) y e) eversión del labio inferior $(3=$ posición labio inferior normal, $2=$ eversión leve, $1=$ eversión moderada, $0=$ eversión severa). Estas mediciones se realizaron en 4 oportunidades: previo a la instalación, en el momento de la instalación y después de los 2 meses de tratamiento con la placa en boca y sin la placa en boca.

Se constató además la postura lingual al dormir, la contención de leche al alimentarse, la emisión de sonidos y la salivación con y sin la placa estimulatoria.

\section{Resultados}

\section{Tono Muscular Labio Superior}

Cuatro de los niños mostraban una hipotonía leve del labio superior en la evaluación de pretratamiento (PrTT), mientras que sólo uno de ellos una hipotonía moderada. En cuanto a la evaluación postratamiento (PsTT) con placa no se observan cambios en la tonicidad del labio superior en tres de los niños, pero si el aumento de un grado en dos de ellos. 


\section{Tono Muscular de la Lengua}

Tres de los niños presentaron una hipotonía lingual leve en PrTT y dos de ellos una hipotonía lingual moderada. Al comparar PsTT sin placa, con PrTT, se observa un aumento de un grado en la tonicidad de la lengua en cuatro de los pacientes, mientras que en uno de ellos se observó una disminución de un grado en la tonicidad lingual. En relación a PsTT con placa, se observa un aumento de un grado en la tonicidad lingual de cuatro niños al comparar los valores obtenidos con PrTT, mientras que en uno de ellos no se observaron cambios.

\section{Postura Labial}

Al momento de PrTT, cuatro de los pacientes presentaron una apertura bucal moderada y uno de ellos una apertura bucal leve. Al comparar los datos obtenidos en PsTT sin placa con PrTT, se vio un aumento de un grado en el cierre bucal en tres de los niños, en uno de ellos éste aumentó en dos grados, mientras que en el otro no se manifestaron variaciones. Cuando se comparan los valores obtenidos en PsTT con placa con las condiciones PrTT se observó un aumento del cierre bucal de dos grados en tres de los pacientes y un aumento de un grado en dos de los pacientes.

\section{Posición de la Lengua}

En PrTT se observó una posición interlabial de la lengua en dos de los niños mientras que los otros tres presentaron una posición lingual interdentaria. En PsTT sin placa, se observó un aumento de un grado en la retracción lingual en cuatro de los niños al compararlos con los valores obtenidos en PrTT, en el quinto niño no hubo cambios en el grado de retracción lingual. Con respecto a PsTT con placa en boca se observa un aumento de un grado en la retracción lingual en tres de los niños y de dos grados en dos de los niños en comparación con PrTT.

\section{Eversión Labio Inferior}

Tres de los niños presentaron una eversión leve de labio inferior y dos una eversión moderada durante PrTT. En relación a PrTT y a PsTT sin placa en boca se observó que no se produjeron cambios en la postura del labio in- ferior en cuatro de los casos, mientras que en uno de ellos se vio un aumento en un grado en la postura labial. Al comparar PrTT y PsTT con placa, se pudo ver que en dos pacientes los valores se mantuvieron, en cambio en los otros tres hubo un aumento de un grado en la postura labial.

\section{Postura Labial al Dormir}

Cuatro niños dormían con la boca abierta en PrTT, y en PsTT, dos niños ya dormían con la boca totalmente cerrada, y dos disminuyeron un poco la apretura. El quinto niño siempre había dormido con la boca completamente cerrada.

\section{Contención de la Leche}

Tres niños botaban parte de la leche cuando la tomaban en PrTT, y en PsTT, dos niños ya no botaban leche, y uno botaba sólo en ocasiones. Los otros dos niños no tenían problemas con la contención de la leche en boca desde antes de la terapia.

\section{Emisión de Sonidos}

Sólo un niño pronunciaba algunas sílabas y palabras cortas antes de iniciada la terapia. Los otros cuatro niños sólo emitían sonidos inentendibles en PrTT, y en PsTT los cuatro niños pronunciaban sílabas y palabras cortas tales como "mamá" y "papá".

\section{Salivación con y sin Uso de la Placa}

Un niño salivaba mucho despierto y en reposo en PrTT, dos salivaban en reposo pero en poca cantidad en PrTT y los otros dos niños no tenían problemas de salivación. Los tres niños que salivaban sin contener la saliva en boca observaron una mejora de su condición en PsTT.

\section{Discusión}

La diferencia en el tono muscular del labio superior y de la lengua durante el tratamiento no es atribuible a la terapia, ya que puede deberse a una contracción momentánea por reconocimiento de un elemento extraño o a un aumento paulatino de la tonicidad previo a la 
terapia, ya que un aumento real del tono sólo se podría evaluar a largo plazo $^{6-8}$.

El aumento del cierre bucal y de la retracción lingual observado en los pacientes durante la instalación puede atribuirse a la denominada "reacción primaria" (cierre bucal completo, y retracción lingual). Los cambios de estos parámetros durante el tratamiento, pueden atribuirse a la constante estimulación de la placa y los ejercicios vibratorios aplicados. Esta observación es coincidente con variados estudios donde se ven cambios a corto plazo en ambas variables. Al ver la similitud de los resultados en el cierre bucal y protrusión lingual, se sugiere una intima relación entre dichas estructuras, pudiendo pensar que la protrusión lingual está influenciando directamente el cierre bucal. Para una ejercitación completa de la musculatura lingual, es necesario estimularla para su retracción y movimientos finos, por esto es necesario incluir cambios en la terapia y en los dispositivos en cada etapa del tratamiento. Además sería útil aplicar dispositivos móviles, para evitar el acostumbramiento a la terapia.

El que no se produzcan cambios en la eversión del labio inferior, puede atribuirse a que está relacionada con la tonicidad del labio inferior, la que sólo cambiara a largo plazo ${ }^{9-12}$.

El aumento del cierre bucal al dormir, podría considerarse como un beneficio de la terapia pero es difícil medirlo objetivamente ya que se ve influenciado por posibles cuadros de obstrucción respiratoria. La mejora en la contención de leche en boca, podría atribuirse al aumento de la tonicidad labial, y al cambio de patrón de deglución que acompaña al desarrollo.

Con respecto a la mejora en la emisión de sonidos, puede deberse a las variaciones en la postura labial y protrusión lingual, aunque también podría relacionarse con la evolución normal del lenguaje, o a la subjetividad de las observaciones de los padres ${ }^{13}$.

La disminución en la salivación, es atribuible a los cambios en la tonicidad labial y postura del labio inferior que permitirían un mejor sellado labial. El aumento de salivación a los primeros días de uso de la placa, se explica como una reacción propia de un proceso de adaptación, que fue disminuyendo hasta lograr una completa contención de la saliva con la placa en boca ${ }^{14}$.

Dentro de las limitaciones del estudio se encuentras el corto período de evaluación, el escaso número de pacientes, la falta de un grupo control y la imposibilidad de determinar la acción neta de las placas ya que se utilizaron en conjunto a los ejercicios de estimulación. Sin embargo, nos pareció interesante dar a conocer esta serie clínica, por los resultados obtenidos a corto plazo.

Finalmente, para obtener un desarrollo integral, el equipo de trabajo debería multidisciplinario, incluyendo: médicos fisiatra, fonoaudiólogos, terapeutas ocupacionales, y odontólogos.

\section{Conclusiones}

La combinación del uso de la placa palatina con los ejercicios de estimulación orofacial produce un aumento del cierre bucal y una disminución de la protrusión lingual, en niños con Síndrome de Down, sin embargo, para cambios en la tonicidad se requieren estudios a largo plazo, y con mas niños, aunque sí podemos decir que la terapia logra mejoras en la expresión facial.

La simplicidad de la terapia, la buena adaptación de los niños y la ausencia de daños, conllevó a una buena adaptación y compromiso de los padres.

La aplicación de esta técnica en nuestro país requiere una implementación, especialización y equipos interdisciplinarios preparados para realizar la terapia.

\section{Agradecimientos}

Dr. Juan Eduardo Onetto, Cirujano Dentista, Profesor Odontopediatría.

\section{Referencias}

1.- Fisher-Brandies H, Avalle C, Limbrock $G$ : Therapy of orofacial dysfunctions in cerebral palsy according to Castillo-Morales: first results of a new treatment concept. Eur J Orthod. 1987; 9: 139-43.

2.- Bäckman B, Grevér-Sjölander AC, Holm AK, Johansson 
I: Children with Down syndrome: Oral development and morphology after use of palatal plates between 6 and 18 month of age. Int J Paediatr Dent 2003; 13: 32735.

3.- Carlstedt K, Dahllöf G, Nilsson B, Modéer T: Effect of palatal plate therapy in children with Down syndrome: a 1 year study. Acta Odontol Scand 1996; 54 : 122-5.

4.- Limbrock G, Castillo-Morales R, Hoyers H, Stover B, Onufer $C$ : The Castillo-Morales a roach to orofacial pathology in Down syndrome. Int J Orofacial Myology 1993; 19: 30-7.

5.- Schuster G, Giese R: Retrospective clinical investigation of the impact of early treatment of children with Down's syndrome according to Castillo-Morales J Orofac Orthop 2001; 62: 255-63.

6.- Carlstedt K, Henningson G, Dahlog G: A four year longitudinal study of palatal plate therapy in children with Down Syndrome: effects on oral motor function, articulation and communication preferences. Acta Odontol Scan 2003; 61: 39-46.

7.- Carlstedt K, Henningsson G, Mc Allister A, Dahlo G: Long-term effects of palatal plate therapy on oral motor function in children with Down Syndrome evaluated by video registration. Acta Odontol Scan 2001; 59: 63-8.

8.- Zavaglia V, Nori A, Mansour N: Long term effects of the palatal plate therapy for the orofacial regulation in children with Down Syndrome. J Clin Pediatr Dent 2003; 28: 89-94.

9.- Hohoff A, Ehmer U: Short-term and long-term results after early treatment with the Castillo-Morales stimulating plate. J Orofac Orthop 1999; 60: 2-12.

10.- Hoyer H, Limbrock G: Orofacial regulation therapy in children with Down Syndrome, using the methods and apliances of Castillo-Morales ASDC J Dent Child 1999; 57: 442-4.

11.- Limbrock G, Fisher-Brandies H, Avalle C: CastilloMorales orofacial therapy: treatment of 67 children with Down Syndrome. Dev Med Child Neurol 1991; 33: 296-303.

12.- Limbrock $G$, Hoyer H, Scheying H: Regulation therapy by Castillo- Morales in children with Down syndrome: primary and secondary orofacial pathology ASDC J Dent Child 1990; 57: 437-41.

13.- Hohoff A, Ehmer U: Effect of the Castillo Morales Stimulating Plate on Speech Development of Children with Down's Syndrome a retrospective study. J Orofac Orthop 1997; 58: 330-9.

14.- Limbrock $G$, Hoyer H, Scheying H: Drooling, chewing and swallowing dysfunctions in children with cerebral palsy: treatment according to Catillo-Morales ASDC J Dent Child 1990; 57: 445-51. 